\title{
EDITORIAL
}

\section{Are You Making Yourself Clear? You Can't Communicate, or Think, Effectively If You Can't Write Clearly}

\section{Curt Tribble, MD}

\author{
Division of Cardiac Surgery, University of Virginia Health System, Charlottesville, Virginia, USA
}

\section{INTRODUCTION}

\section{I write for discovery.--Joe Henry, songwriter [NPR 2014]}

In the not too distant past, illegible handwriting was considered to be the biggest problem with medical record keeping. Now the primary problem with medical records is that they are disorganized, and usually undigested, data dumps. A solution to at least part of this problem lies in utilizing the principles of the problem-oriented record.

When one contemplates the optimal format for progress notes, it is worth considering the purposes of progress notes. While progress notes do, of course, play a role in billing, the primary purposes of a progress note should be to provide efficient and effective communication with all who are caring for that patient and to facilitate efficient and effective contemplation of the condition of and the plans for that patient. Although it is beyond the scope of this treatise on creating progress notes, it is also worth pointing out that all patient care notes will also occasionally have legal implications and lawyers reading clinical notes will pay far more attention to assessments and plans than they will to data and results recorded in progress notes that are always easily available elsewhere in the patient record. In other words, lawyers reviewing medical records want to know what the clinicians caring for a patient were thinking, in addition to what those clinicians actually did for that patient.

While all of these issues must be kept in mind, we will focus primarily on the role of clinical notes in providing optimal patient care, particularly in the realm of cardiothoracic surgery, though the principles to be enunciated can apply to most disciplines and to most clinical environments.

\section{THE HISTORY OF THE DEVELOPMENT OF THE} PROBLEM-ORIENTED RECORD

The unaided human mind is not a reliable instrument for the processing of information in the solution of patients' problems. It should not be licensed to try the impossible. Yet medical education encourages physicians to try exactly that. - Lawrence Weed, MD

Correspondence: Curt Tribble, MD, Professor of Cardiothoracic Surgery, University of Virginia Health System, Division of Cardiac Surgery, Box 800679, Charlottesville, VA 22908; 434-243-9250 (e-mail: ctribble@virginia.edu).
The problem-oriented record was first described by Dr. Lawrence Weed, an "engineer turned internist" who practiced and taught at the University of Vermont in the late 1960s. His approach to record keeping was, for decades thereafter, called "The Weed Problem-Oriented Medical Record." However, his descriptions of this strategy were felt by many to be a bit long-winded to the point of being, perhaps, somewhat pedantic [Weed 1968]. Dr. J. Willis Hurst, Chair of Medicine at Emory for at least a generation (and the personal physician of President Lyndon Johnson), passionately and effectively championed Dr. Weed's method [Hurst 1971]. One of Dr. Hurst's essays on this subject was entitled "Garbage in the Living Room" [Hurst 1995]. In this humorous essay, Dr. Hurst describes a scenario of being invited to someone's house for dinner and arriving to find the living room strewn with garbage, chicken bones, and empty cans. He notes that you would not be able to know with certainty whether the rest of the house was also strewn with similar debris but that it would be safe to assume that it was. He uses this colorful analogy to make the point that, if your medical records are messy and disorganized, it is not certain that your thoughts are similarly disorganized, but it is a pretty sure bet that they are. I first read Dr. Hurst's defenses of Dr. Weed's system when I was a medical student [Zuidema 1974]. I took the liberty of writing Dr. Hurst a letter to say how much I liked his description of this imminently sensible approach to medical record keeping. To my amazement, he wrote me back. And, thus began a 40-year relationship, maintained at first by the exchange of notes and later through the use of e-mail. I received my last e-mail from Dr. Hurst just a few days prior to his death when he was in his mid-90s [Tribble 2016b].

\section{THE UNEVEN ADOPTION OF THE PROBLEM- ORIENTED RECORD}

However, despite support from well-known academicians like Dr. Hurst, Dr. Weed's approach to medical record keeping has been only partially adopted by the medical community. For example, many use the so-called "SOAP Note" format, but most practitioners do not use this format in the true problemoriented style described by Dr. Weed and promoted by Dr. Hurst.

Most students, residents, and practitioners know that the SOAP acronym stands for subjective, objective, assessment, and plan. However, most who use this method of organizing their notes do not do so with a full understanding of what should be included in each aspect of these notes. 
Subjective means "history," which is not restricted to quotations from the patient. It should contain any historical information that has been gathered, whether from the patient, the family, other health care providers, or even from older records.

Objective is a bit confusing to many, because it should encompass both the physical exam and data from lab values, radiologic exams, or other studies.

Assessment, luckily, means just what that word implies, which is that this section of a note should include the reflections of the author of the note on the data presented.

And, Plan, similarly, is supposed to be the outline of what will, or might be, done in response to the assessment of the data presented. However, where the vast majority of progress note writers get off the track is to fail to recognize that there should be a SOAP note (in essence, a short version of a history and physical exam) for each problem, not for each patient, covering a specific day of that patient's hospitalization.

\section{SMART (AND ORGANIZED) BREVITY}

The presentation of irrelevant medical data may be useful for a junior student ... but the persistence of such a babit throughout the years impedes the development of logic. - J. Willis Hurst

Most students and residents create a "data dump" when writing progress notes, and they are prone to do the same when presenting their patients on rounds. When presented with a large amount of disjointed information, those who are reading these notes or listening to these presentations are very often nearly hypnotized. Frequently, these notes or presentations contain disjointed information and include a fair amount of unnecessary data. One must learn to organize information about a patient into a coherent system, to present it in that way, to write about it in that way, and, most importantly, to think about it in that way.

I am not here to teach you facts. If you want facts, read my book. I am here to teach you bow to think. -Tinsley Harrison, MD, author of Harrison's Principles of Internal Medicine

Presenting patient information (in a note or in a verbal presentation) as a "data dump" is analogous to handing the reader or listener a Rubik's Cube with the colors all mixed up:

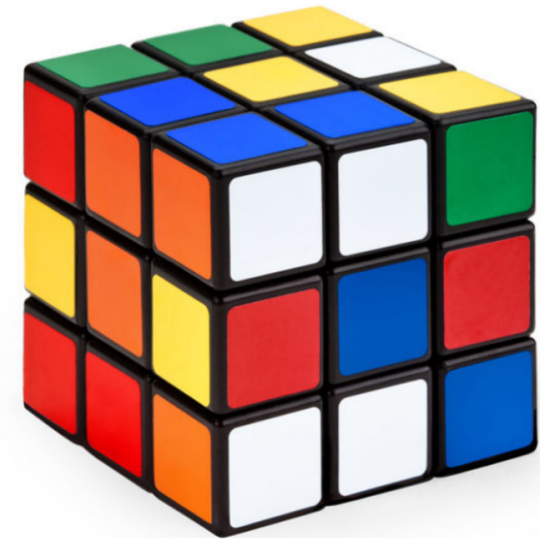

On the other hand, writing or presenting with the problem-oriented method is like handing the reader or listener a Rubik's Cube with the colors all aligned:

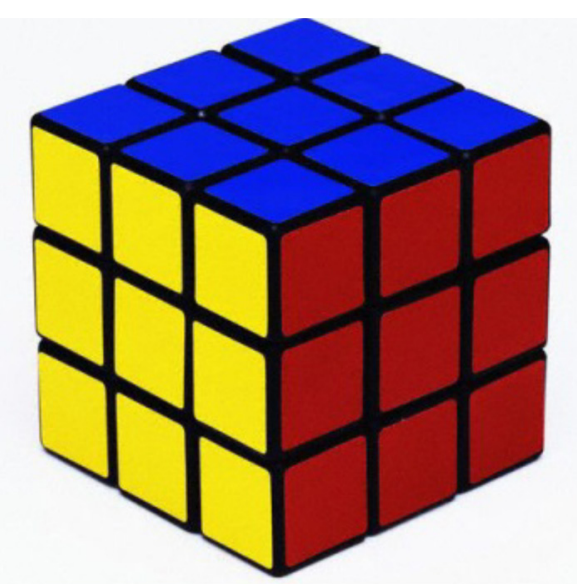

If you were in charge of a patient and were trying to make appropriate decisions about that patient, which type of presentation would you prefer (whether oral or written)? And, which type of presentation would facilitate optimal thinking and decision-making?

\section{CREATING A PROPER PROBLEM-ORIENTED} PROGRESS NOTE

The Problem-Oriented medical record reflects an orderly process of problem solving, a beuristic that aids in identifying, managing, and resolving patients' problems. - The Institute of Medicine, Computer-Based Patient Record, 1991

The first step in creating a problem-oriented progress note is to define which problems a particular patient has. Obviously, the next step is to organize all the available information about the patient according to that problem list. Disorganized information is a recipe for confusion at best, disaster at worst.

Most of our cardiothoracic patients have 3 to 5 main problems, besides the issue for which we operated on them. Keep a list of those problems on an index card that you keep with you at all times. Don't ever let a patient presentation conclude without addressing each of those problems. - Joe Baker, MD, Cardiothoracic Resident, University of Virginia, 1989

Usually the patients on typical cardiac or thoracic surgical services have 3 to 5 main problems, in addition to the primary issue for which they have been admitted. You should always be able to cite the main problems you are concerned about in each patient. Some patients will have fewer problems, but the average number of problems is about 4. On a daily basis, you should gather data, write about that data, present that data, and, above all, think about that data, as they relate to your patients, organized by those problems. It is generally best to keep the order of the problems the same 
each day to help you, and everyone on your team, remember what the patients' issues actually are.

\section{EFFICIENT MANAGEMENT OF MEDICAL INFORMATION, DAY TO DAY}

I'm not writing it down to remember it later, I'm writing it down to remember it now. - Field Notes website

Once a clinician has created a note in the problem-oriented format, cutting and pasting it forward (with appropriate updates) is not only reasonable but is also, obviously, both efficient and effective. That efficiency and effectiveness, of course, involves being forced to consider, in turn, each of the patient's problems on a daily basis.

A useful strategy can be to print out your daily progress note, once it is complete, and to use it during the next day as you gather information on each patient throughout that day, leading up to creating the updated progress notes for that day. Furthermore, you can use such notes to present on rounds, even if they are still "a work in progress." For instance, you may need to present a patient on rounds to find out the final plans for that patient prior to putting that day's note into the electronic medical record (EMR), particularly if you are a relatively junior member of the team.

\section{WRITE ONLY MEMORY (WOM) VERSUS READ ONLY MEMORY (ROM)}

Your priority should be to create notes that are both easy and efficient for others to read and follow. The goal should be to create notes that allow the readers of your notes to get the gist of the note "in a glance," while still being thorough. Here are some suggestions on ways to help accomplish these goals:

- A brief introduction is helpful.

- A short summary of the events of the last 24 hours at the beginning of the note can be useful, as well.

- And, a brief summary, particularly of the patient's current issues and plans, at the end of the note, can also be helpful to readers of your note.

\section{A BRIEF MANUAL OF STYLE FOR PROGRESS NOTE WRITING}

Here are some additional tips for efficient and effective note writing:

Make everything you write as "telegraphic" as practical.

Do not cut and paste full reports into the note. Summarize all information tersely. This process will not only reflect accurate reading of these reports by you but will also make reading these summaries efficient for subsequent readers.

It can be helpful to cut and paste these reports into your note and then take a few moments to edit them to make them as terse as feasible.

Consider putting only data pertinent to a specific problem in that problem's "section." That strategy will allow you to keep repetition to a minimum in the note as a whole.
Use abbreviations judiciously. That is, use only common ones.

Consider using pluses (+) and minuses (-).

Here's an example for the exam section of a note, describing the problem of fluid overload:

+ : rales, most prominent in left lung base

-: no ankle edema

(This strategy is very efficient for both the writer and the reader.)

Vital signs are part of the physical exam.

(Include only the vital signs pertinent to a specific problem in the section dedicated to that problem. For example, record the blood pressure under "hypertension," and record the maximum daily temperature under "infectious disease.")

Similarly, list individual lab values under the problem to which they are related. For example, list the hematocrit under anemia and the BUN and creatinine under fluid overload or renal dysfunction, depending on which problems the patient actually has.

(Volume status is a problem for virtually every cardiac and thoracic patient. Therefore, "volume status" should be listed as a problem for almost all these patients.)

Distinguish between "assessment" (what you think is going on) and "plan" (what you think you are going to do about that assessment). These sections of your note should be 2 sequential sections, under each problem listed. (And, remember that it is okay to list "tentative" plans or plans that are "being considered," because, especially if you are a relatively junior member of the team, you may frequently be writing your note prior to the plans being finalized.)

Deciding where to list medications in your notes is a bit tricky. Some like to list them all together, perhaps at the beginning or the end of the note. My own instinct (usually, at least) is to list the medications under the problem that they are related to. For instance, you can list Lasix under volume status and beta-blockers under cardiac rhythm or hypertension. However, the manner in which you list the medications in a progress note does not relieve you from the obligation to review the medication administration record (MAR) each day to see what meds the patient was actually given, as this list can differ from what has been ordered. In fact, a standard line in a progress note could be: "The MAR for the last 24 hours was reviewed" or something to that effect.

It is desirable to make the plans that you list track, point by point, with the assessment. (That is, keep the assessment and plans in the same order under each problem). It is certainly easier to read and follow these 2 parts of your note when presented in this way.

Avoid using the word "endorse." It's just weird. One endorses a political candidate. Just write "patient c/o pain," rather than "patient endorses pain." (And, this shorter phrase takes less time to type, as well!)

Try not to list too many lab values on one line.

(And, try to make these entries as terse as possible).

Do this: prealbumin: 29 on 2-28 (normal: $18-45$ ) albumin: 3.2 on 3-6 (normal: 3.5-5.5) 
Not this:

prealbumin on 2-28 was 29

albumin on 3-6 was 3.2

See how much easier the first style of presentation is to read and to contemplate?

For a terse outline of a plan...

Write this:

POD 5 - Ba swallow. Start clears if normal.

Instead of this:

Order Ba swallow study for POD 5 and consider progressing diet to include clear liquids if the swallow is normal.

\section{WHAT SHOULD BE INCLUDED IN A PROBLEM- BASED CARDIOTHORACIC SURGERY PROGRESS NOTE (AND WHY)}

I have long thought that I wanted to know why I should be doing something that I was told to do. In this section, I will review, in detail, which issues should be included in a progress note about a cardiothoracic surgery patient, why these issues need to be addressed, with a suggested format for them.

What: A list of the patient's problems.

Why: To group data points to optimize decision-making, to lessen the monotony of progress notes or presentations, and to avoid neglecting important issues.

What: Mr. is POD \# is hospital day \# from a . (Or Ms.

Why: We organize our thoughts about a patient and his or her progress around the operation that was done and how far out they are from the operation or initial day of hospitalization. It is hard to do the math on a patient's trajectory by merely citing dates. of it.

What: The patient's fluid status and our management

Shortness of breath?

Weight (always compared to the pre-op weight).

Urine output (UOP). I's and O's.

PE: rales? edema? (Look at flanks, sacrum, hands, face, and ankles.)

Hemodynamics: CVP (central venous pressure) or PAD (pulmonary artery diastolic pressure).

Labs: BUN, sodium.

Oxygenation: $\mathrm{PO} 2$ or $\mathrm{O} 2$ saturation.

Chest $\mathrm{x}$-ray (CXR) appearance.

Diuretic Rx.

Why: The most consistent and often the most important issue in our cardiac and thoracic patients is their fluid status. They are virtually all fluid overloaded. When they are "dry," they can go home. If they are "wet" at discharge, they will often return to the hospital and, frequently, be readmitted. The challenge includes figuring out intravascular volume status versus total body volume status, as patients can have a low intravascular volume, while still having excess fluid in their tissues.
What: Pulmonary function.

Respiratory symptoms, oxygen saturation, incentive spirometer, supplemental oxygen use, CXR, secretions, respiratory treatments.

Why: This issue is a close second to (and closely related to) fluid status in determining when a patient can move to the floor from the intensive care unit (ICU) or when the patient can go home.

What: Cardiac rate and rhythm.

Sinus rhythm $=$ good. Atrial fibrillation $=$ bad.

Rate (beats per minute): $60-80=$ good. $80-100=\mathrm{OK}$. Over $100=$ bad.

Why: Cardiac rhythm will often be the rate limiting steps in post-op progression if fluid status and oxygenation are not the rate limiting steps.

What: Cardiac function.

Cardiac index if a Swan Ganz catheter is in place. Echo if done postoperatively. Preoperative function is considered in determining the medications needed now and at discharge, as well as the need for follow-up.

Why: Must sort out what needs to be done to optimize cardiac function in the short- as well as the longterm. Discharge medications for heart failure patients or atherosclerotic patients are monitored for quality control.

What: Renal function.

UOP, BUN and creatinine. History of renal dysfunction preoperatively, diuretics administered.

Why: Important for prognosis and for short-term decision-making (dialysis, diuretics, avoiding nephrotoxic drugs, managing fluid status, etc.).

What: Tubes, lines, Foley catheter, wires, nasal cannulae. How long have they been in place? What can we get out or discontinue? Have you documented reasons for leaving them in? (Central lines and Foley catheters, especially.)

Why: We need to aggressively remove every catheter and tube that is no longer needed in order to avoid infection, to increase mobility, and to pass muster with quality control systems. (A Foley catheter must be removed by POD \#2 or you must document reasons for leaving it in. The need for CVP lines also must be documented daily.) Supplemental O2 causes atelectasis and "ties patients down." (As an aside, it is worth noting that chest tubes virtually never cause infection.)

What: Glucose.

Glucose should be below $200 \mathrm{mg} / \mathrm{dL}$ on POD \#1 and POD \#2. What treatment is the patient receiving for hyperglycemia? What is the pre-op history of glucose intolerance and treatment?

Why: Glucose control is also monitored as a quality measure, because control of hyperglycemia lessens infection risk and can increase cardiac function. 
What: Infection control.

Maximum temperature each day, antibiotics administered, white blood cell count with bands, culture results, potential sources of infection.

Why: Many patients have some potential infectious issues or are being actively treated for infection.

What: The other 2 or 3 issues that most patients will have but that are not covered in the list above.

(These other issues include medical issues, such as gout, wounds, psychiatric issues, alcohol withdrawal, glaucoma, debility, etc.) List the specific issues for each patient each day, even if not much is going on with them, to remind all on the team, as well as all consultants, of these issues.

Why: Every patient has a handful of issues that we must pay attention to in addition to his or her primary reason for hospitalization.

What: Medications.

List medications, such as diuretics, blood pressure medications, statins, and anticoagulants (as well as plans for followup for those on Coumadin, as time for discharge approaches). As noted earlier, it is also reasonable to list these meds in the note under the problem for which they've been prescribed.

Why: Most patients with cardiothoracic issues need to be on some or all of these medications. Most need to have at least a beta-blocker, anticoagulation of some sort (ASA, Plavix, or Coumadin), angiotensin converting enzyme (ACE) inhibitors (for decreased cardiac function), and diuretics (with potassium). Quality measurement systems monitor compliance with adherence to prescribing these medications, when they are indicated.

\section{Addendum}

While this treatise is about progress notes, it is worth remembering that when creating a note for an initial encounter (history and physical exams, consults, initial clinic visits, etc.), one can record, perhaps in the social history, a note about what the patient would like to do when he or she is well again. Besides reminding yourself of this plan, recording this information can be valuable to many others who will participate in the patient's care [Tribble 2016a (Gimme 3 Steps)].

\section{ICU NOTES}

Many intensivists prefer that notes about patients in ICUs be created in an organ system-based format rather than a problem-oriented format. The reason for this preference is that these patients are so complicated (or at least potentially complicated) that the organ system-based format may be the best way to ensure the contemplation and the recording of all of the issues of very complex patients. Thus, the problemoriented format may be optimally reserved for patients still in an intensive care unit but who are not critically ill [Thaemert 2009 (ICU Recall, 3rd edition)]. That is, some intensivists believe that, once an ICU patient has stabilized or can be considered a more chronic patient, a problem-oriented approach to record keeping can be used.

\section{ELECTRONIC MEDICAL RECORDS}

Among the many complaints about electronic medical records (EMRs), the death of the useful medical note ranks very high.

... It's often impossible to glean what a clinician thinks is

going on, or what's planned. ...

... The medical words are there, but where is the content? Paul E. Sax, MD [Sax 2019]

Many practitioners, including Drs. Weed and Hurst, decades ago, envisioned that computerized medical records would be an enormous "leap forward" in medical record keeping. However, the computerized medical record has not created unmitigated bliss for medical practitioners. Far from it, in fact. Four decades after Drs. Weed and Hurst were promoting the use of the problem-oriented record and predicting that a computerized record would save practitioners from the inefficiencies of writing by hand and of reading often illegible handwritten notes, nearly everyone agrees that we are now mired in a different kind of mess. Moreover, as Dr. Abigail Zuger noted in a recent post: "The more detailed and comprehensive is the note, the smaller are the chances that any of the listed details were actually elicited or that the described activities EVER took place, even in their first iteration" [Sax 2019]. Many have described these long, cumbersome, and often inaccurate notes as "note bloat" [Versel 2014]. In fact, progress notes and consultations in the United States are, on average, nearly 4 times longer than those in other countries, which obviously makes them much less efficient both to create and to read [Sax 2019].

What are the potential solutions to these problems with the EMR?

First of all, the writers of these notes must take ownership of their work. They need to think critically, carefully, and thoroughly about every word and every data point that they include in their notes. While autofill, dot phrases, and macros can be time savers in creating medical notes, the authors of these notes simply must take pride and ownership of the notes that they construct and sign. That is, they need to become artisans skilled in the craft of good medical record keeping. Specifically, those who write these notes must take the time to proofread their work, with the following goals:

- Making them as efficient to read as humanly possible

- Ensuring that the data are accurate at the time the note is created

- Reflecting on the information presented

- And, outlining a plan, based on the available information and the reflections on that information. And, if that plan has to await approval by others, that fact can be simply stated in the note.

Second, the authors of these notes in an EMR could consider using templates of their own to facilitate efficient data entry and organization. For example, as I myself became familiar with using an EMR in recent years, I realized that I could make efficient and effective use of templates not only for progress notes but also for clinic or consultation notes as 
well as for operative notes. These templates can be saved as Word documents, complete with the 3 asterisks that will prevent one from signing the note, if the proper data have not yet been entered into the note at certain points. It does not take long to build up a library of these very useful templates.

Third, it is worth noting that importing key results into a template yourself, rather than having these results "autofill" the note, will help ensure that these imported data have actually been contemplated by you, the author of that note. Furthermore, inserting these key results does not actually take all that much time.

Fourth, it is useful to consider how a note of any type reflects on you as a craftsperson, as a careful thinker, and as a clinician who is not only taking charge of the patient's care but who is also dedicated to making sure that all others involved are thoroughly and accurately informed about the patient's critical issues.

Finally, it is worth noting that there are some creative people thinking of improvements to electronic medical records that might make them even more useful and efficient. One such strategy has been proposed by Edward Tufte, a renowned expert on the visual display of quantitative information. In an article in The Lancet, Dr. Tufte and a physician colleague at Yale propose a method of presenting a graphical summary of a patient's hospitalization, which allows the reader to understand the course of a quite complex patient on a single page [Powsner 1994].

\section{CONCLUSION}

If you can't explain it simply, you don't understand it well enough. -Albert Einstein

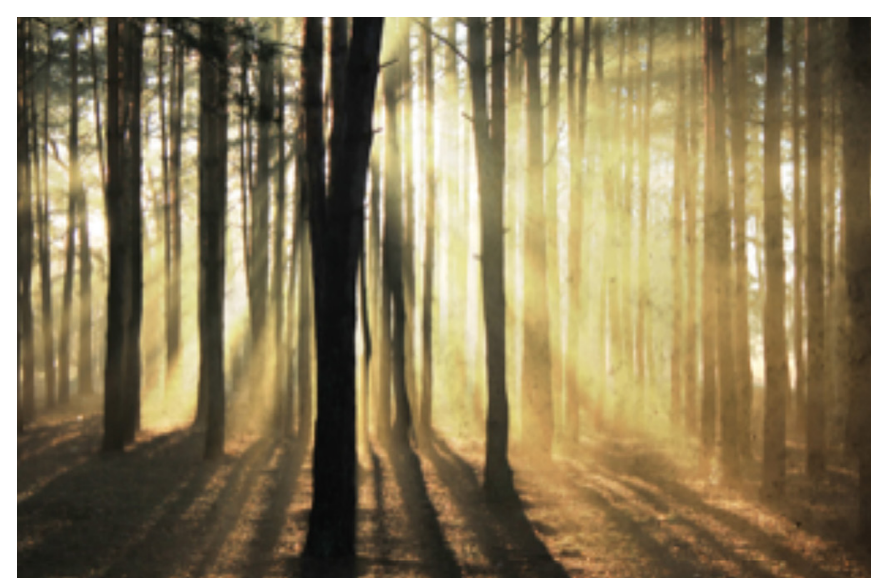

Photo by Valeriy Andrushko. Accessed June 18, 2019. Available from Unsplash at https://unsplash.com/@voodushevlyonniy.

In summary, you need to be able to see, and to help others to see, both "the forest and the trees," by creating thorough, well organized problem-oriented progress notes, which are essential to providing optimal care to your patients. .

\section{REFERENCES}

Field Notes. [accessed 2019 Jun 16]. https://fieldnotesbrand.com/from-seed.

Hurst JW. 1971. Ten reasons why Lawrence Weed is right. New Engl J Med 284:51-2.

Hurst JW. 1995. Essays from the heart. New York: Raven Press. Garbage in the living room; p. 136-9.

Institute of Medicine. 1991. Computer-based patient record: an essential technology for health care. Washington (DC): The National Academies Press; [accessed 2019 Jun 10]. https://doi.org/10.17226/18459.

National Public Radio (NPR) Staff. Joe Henry on marriage and the songs that embody it [music interview]. Weekend Edition Sunday. NPR. 2014 Jun 29, 11:40 minutes. [accessed 2019 Jun 10]. http://www.npr.org/2014/06/29/325530723/ joe-henry-onmarriage-and-the-songs-that-embody-it.

Powsner SM, Tufte ER. 1994. Graphical summary of patient status. Lancet 344:386-9.

Sax PE. 2019 Jan 2. How did our medical notes become so useless [blog]? HIV and ID Observations. [accessed 2019 Jun 10]. https://blogs.jwatch.org/hiv-id-observations/index.php/ how-did-our-medical-notes-become-so-useless/2019/01/02/.

Tribble C. 2016. Gimme 3 steps (with a nod to an American rock song from the 1970s). Heart Surg Forum 19:E080-1.

Tribble C. 2016. On becoming a doctor of humane letters. Heart Surg Forum 19:E150-2.

Thaemert NL, Hobson CE, Tribble CG, eds. 2009. ICU recall. 3rd ed. Philadelphia: Lippincott Williams \& Wilkins.

Versel N. 2014 May 8. Physicians rethinking the progress note: physicians assess 'SOAP' vs. 'APSO.' Healthcare IT News (Global Ed.). [accessed 2019 Jun 14]. https://www.healthcareitnews.com/news/ rethinking-progress-note.

Weed L. 1968. Medical records that guide and teach. N Engl J Med 278:593-600.

Zuidema G, Judge R. 1974. Methods of clinical examination: a physiological approach. 3rd ed. Boston: Little Brown \& Co. 